\title{
Photonic clocks, Raman lasers, and Biosensors on Silicon
}

\author{
K. Vahala, T. Carmon, H. Roksari, T. Kippenberg, A. Armani, D. Armani \\ MS 128-95 California Institute of Technology, Pasadena, CA 91125 \\ vahala@caltech.edu
}

\begin{abstract}
Micro-resonators on silicon having Q factors as high as 500 million are described, and used to demonstrate radio-frequency mechanical oscillators, micro-Raman and parametric sources with sub-100 microWatt thresholds, visible sources, as well as high-sensitivity, biological detectors.
\end{abstract}

There are numerous applications resulting from ultra-high-Q performance in microresonators, including cavity QED and passive filters $[1,2]$. In this paper we will describe a set of emergent nonlinear phenomena including: the first examples of micro Raman [3] and OPO [4] devices on a chip, visible third harmonic sources [5], as well as a novel device that couples optical power to resonator mechanical motion via radiation pressure [6]. In each of these applications, high-Q serves to time-integrate optical power coupled to the whispering gallery, while the microscale mode volume concentrates this power and thereby creates high circulating field intensities. Finally, we will also discuss the application of these devices as sensors for both chemical and

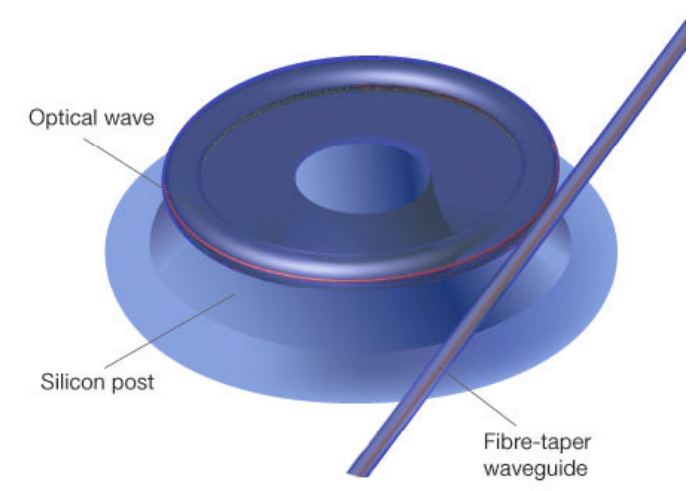

Figure 1: Ultra-high-Q toroidal micro-resonator coupled to a silica-fiber taper waveguide. The toroid is typically 30 microns in diameter.

biological detection.

The resonator device is illustrated in figure 1 and is in the form of a microtoroid [7]. Being a silica-on-silicon device, it provides many of the inherent advantages of other wafer-based microresonator structures. In particular, it leverages the linewidth control and process parallelism of semiconductor wafer fabrication methods. Significantly, however, a unique, silica reflow step endows toroidal whispering-galleries with a liquid-like smoothness that dramatically reduces surface scattering and hence produces ultra-high-Q. To date, Q factors as high as 500 million have been demonstrated using these devices. At the time of their demonstration this represented a 5000-fold improvement in Q factor over all prior wafer-based devices [7].

Raman oscillation occurs readily in these structures at sub-100-microWatt threshold power levels [3]. Figure 2 provides an output power versus pump power plot for near threshold operation. Well above threshold, the Raman process can excite many longitudinal modes of the device. Raman gain belongs to the stimulated class of nonlinear phenomena, however, the material silica can also exhibit third-order parametric nonlinearities. Until recently, parametric effects in micro-resonators have been elusive because, in addition to high field intensities, they require phase matching of pump,

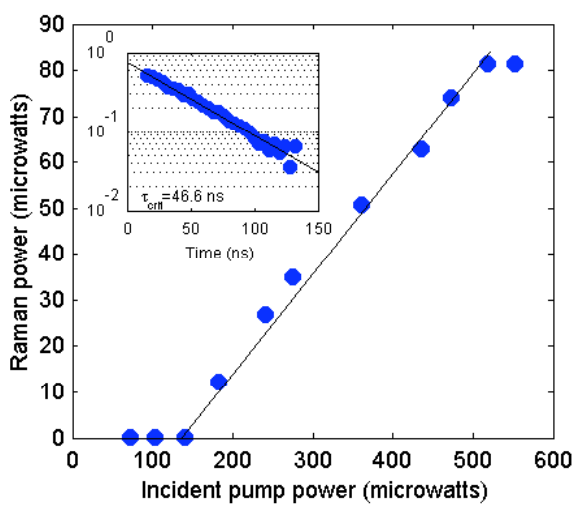

Figure 2: Near threshold output power versus pump power plot for a Raman laser illustrating threshold of 140 microWatts.

signal and idler waves. Remarkably, the toroid geometry enables control of phase matching through control of the toroid "aspect ratio" (the ratio of toroidal major to minor diameters). By adjustment of this ratio, a transition from Raman oscillation to parametric oscillation has been demonstrated [4].

In addition to Raman and Parametric oscillation, the third-order nonlinearity also can be used for third harmonic generation [5]. In this case, the existence of higher-order spatial modes 
within the toroid allows the phase-matching of third-harmonic emission as shown in figure 3 where an IR wave in the $1500 \mathrm{~nm}$ band is used to excite several colors in the visible band [5].

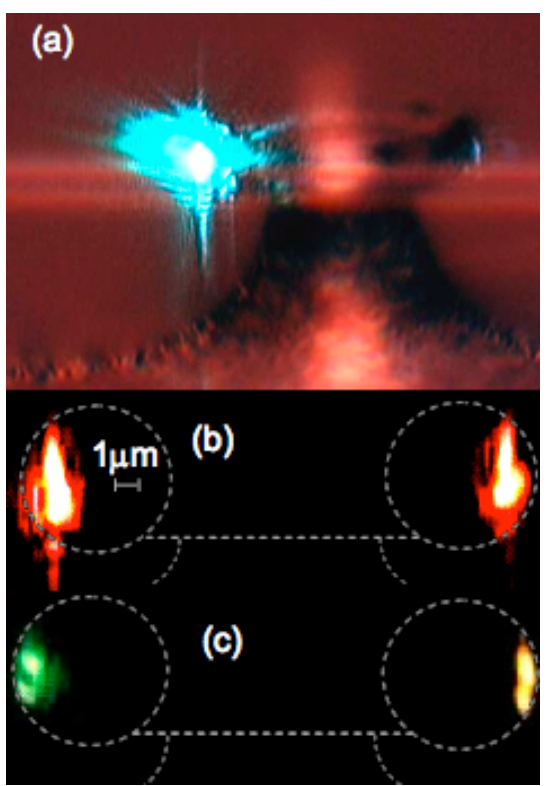

Figure 3: Visible third harmonic emission from a microtoroid. Panel (a) shows blue emission from a single pump in the $1500 \mathrm{~nm}$ band while panels $(\mathrm{b})$ and (c) show multiple colors using several pumps.

In addition to purely-optical nonlinear phenomena, as described above, toroids also exhibit a entirely new nonlinearity linking optical and mechanical degrees of freedom [6]. High circulating optical power within the toroidal whispering gallery leads to radiation pressure that is sufficient to stretch the diameter the silica structure. This, in turn, modifies the optical path length of the resonant mode, changing its resonant frequency. Under appropriate conditions an accompanying dynamic unfolds that results in regenerative oscillation of the toroidal micromechanical structure at radio-frequency rates. These micro-mechanical oscillations, in turn, modulate the optical power of the incident pump wave. This modulation provides a convenient means by which the oscillations can be studied. A rendering of this process is provided in figure 4 . These devices have been referred to as photonic clocks because they function much like a conventional quartz oscillator except where optical power, as opposed to electrical power, drives the oscillation. Details on the mechanical modes as well both sub- and above-threshold operation of the mechanical oscillator will be discussed including recent observation of microwave rate oscillations.
Finally, high-Q microcavities have demonstrated very sensitive detection of biological molecules [8]. A limiting factor in these previous experiments was the linewidth or $\mathrm{Q}$ factor of the resonant cavity. Recently, toroidal resonators demonstrated Q's greater than 100 million in an aqueous environment [9]. These ultra-high quality factors will enable ultra-sensitive biological and chemical detection. We will conclude by reviewing recent progress in this important direction [10].

1) K. J. Vahala, Optical Microcavities,. Nature 424, pp. 839-846 (2003).

2) K. J. Vahala (ed.), Optical Microcavities,. (World Scientific Publishing, Singapore, 2005).

3) Kippenberg, T. J., Spillane, S. M., Armani, D. K. \& Vahala, K. J. Ultra-low-threshold Raman laser on a microelectronic chip. Optics Letters, vol 29, no. 11, June (2004).

4) Kippenberg, T. J., Spillane, S. M. \& Vahala, K. J. Kerr nonlinearity optical parametric oscillation in an ultra-high-Q toroid microcavity. Physical Review Letters, vol 93, no. 8, August (2004).

5) T. Carmon, K. Vahala, CLEO, Long Beach (2006).

6) H. Roksari, et. al. Radiation pressure micro-mechanical oscillator, Opt. Exp. 13, July 2005; T. Carmon, et. al. Phys. Rev. Lett. 94, June 2005.; T. Kippenberg, et. al. Phys Rev. Lett. 95, July 2005.

7) Armani, D. K., Kippenberg, T. J., Spillane, S. M. \& Vahala, K. J. Ultra-high-Q toroid microcavity on a chip. Nature $\mathbf{4 2 1}$, 925-928 (2003).

8) S. Arnold, M. Khoshsima, I. Teraoka, S. Holler, and F. Vollmer, "Shift of whispering-gallery modes in microspheres by protein adsorption," Optics Letters, vol. 28, pp. 272-274, 2003.

9) A. M. Armani, D. K. Armani, B. Min, K. J. Vahala, and S. M. Spillane, "Ultra-high-Q microcavity operation in $\mathrm{H} 2 \mathrm{O}$ and D2O," Applied Physics Letters, vol. 87, 2005.

10) A. M. Armani, Vahala, K. J., "Heavy water detection using ultra-high-Q microcavities," Optics Letters, vol. 31, 2006.

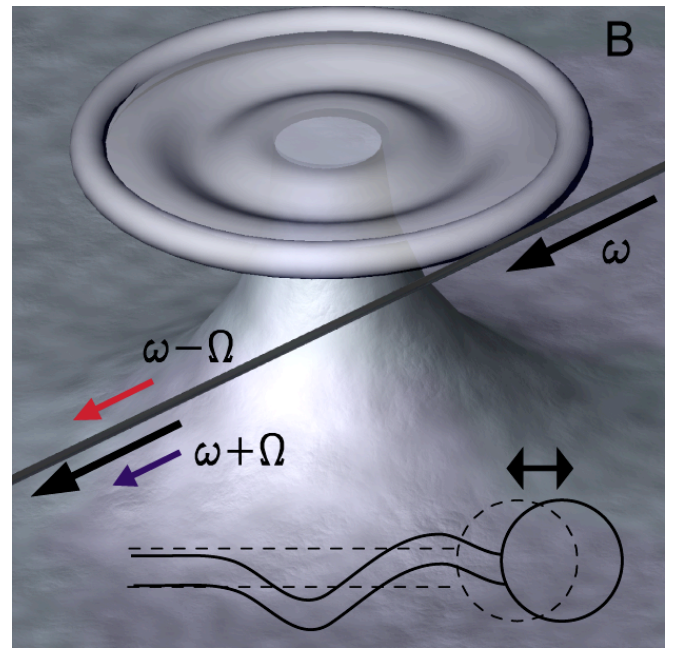

Figure 4: Rendering of regenerative radio-frequency mechanical oscillations of a microtoroid driven by continuous-wave optical pump [6]. 